\title{
Relieving Effect of Quercetin on Ischemia/Reperfusion- Induced Liver Damage in an Animal Model
}

\author{
Toru Shizuma* \\ Department of Physiology, School of Medicine, Tokai University, Japan
}

Received: December 28, 2013; Accepted: February 10, 2014; Published: February 12, 2014

*Corresponding author: Toru Shizuma, Department of Physiology, School of Medicine, Tokai University, Isehara, Kanagawa 259-1193, Japan, Tel:+81-0463-93-1121; Fax:+81-0463-93-6684; E-mail: shizuma@is.icc.u-tokai.ac.jp

\begin{abstract}
We examined the effect of quercetin in relieving ischemia/ reperfusion-induced liver damage using an animal model. F344 rats were divided into a control group ( $\mathrm{n}=8$ ) and a group administered $100 \mathrm{mg} / \mathrm{kg}$ of quercetin (quercetin-treated group) (n=8). Both groups underwent laparotomy, and the portal vein was ligated for $60 \mathrm{~min}$ to simulate ischemia. After 60 min of reperfusion, serum aspartate transaminase (AST)/alanine aminotransferase (ALT) levels, tumor necrosis factor-alpha (TNF- $\alpha$ ) levels, and blood nitrotyrosine yield were measured. The livers were also excised to examine histology. Our results showed that AST/ALT levels, TNF- $\alpha$, and nitrotyrosine yield were all significantly lower in the quercetin-treated group than in the control group. Liver damage was also significantly reduced histologically. Based on these results, administration of quercetin relieves ischemia-reperfusion-induced liver damage.
\end{abstract}

Keywords: Quercetin; Ischemia-reperfusion; Antioxidant effect; Nitrotyrosine

\section{Introduction}

Ischemia/reperfusion (I/R)-induced liver damage can be a problem in surgeries such as liver resection and liver transplantation [1-3]. I/R-induced liver damage can include liver tissue damage and microcirculatory disturbance caused by the production of reactive oxygen species (ROS) through the activation of Kupffer cells, hypercytokinemia, and protease through activated neutrophils [2-5].

Quercetin is a flavonoid found in fruit and vegetables that has a strong antioxidative stress effect through the scavenging of free radicals [4-8]. Its protective effect with regard to post-I/R organ damage has been reported for various organs, such as the heart [9], brain [7], kidneys [10], and gastrointestinal tract, and its antioxidant effect is considered to be the primary mechanism of action $[4,6,7,10,11]$. Here, we examine the effect of quercetin, which has a strong antioxidative stress effect, on I/R-induced liver damage using an animal model.

\section{Materials and Methods}

\section{Experimental animal model and drug}

Eight-week-old male F344 (Fisher) rats (CLEA Japan Inc.,
Japan) were reared on a specific pathogen-free diet and randomly divided into two groups ( $\mathrm{n}=8$ for each). The control group was reared on the standard CE-2 rodent diet (CLEA Japan Inc., Japan) without quercetin, while the quercetin-treated group was reared on the CE-2 diet with quercetin (Sigma, USA). Quercetin was dissolved in distilled water and injected intraperitoneally (100 $\mathrm{mg} / \mathrm{kg}$ ) $24 \mathrm{~h}$ before the I/R experiment. Both groups were fasted for $12 \mathrm{~h}$ before the experiment.

\section{Methods}

Both groups underwent laparotomy under inhalation anesthesia with isoflurane (Wako Pure Chemical Industries, Ltd., Japan) to expose the main trunk of the portal vein. The entire circumference of the portal vein was pinched with a clamp and ligated (ischemia) for 60 min after which the clamp was removed to reperfuse the organ. Blood was collected $60 \mathrm{~min}$ after completing the ischemia, and serum aspartate transaminase (AST)/alanine aminotransferase (ALT) levels, tumor necrosis factor (TNF)- $\alpha$ (ELISA), and blood nitrotyrosine yield [highperformance liquid chromatography (HPLC)] were measured. In addition, after rats were sacrificed, the livers were excised to examine liver histology. Histological examination involved fixing the samples with $10 \%$ formalin, embedding them in paraffin, and performing hematoxylin-eosin (H \& E) staining.

\section{Statistical analysis}

ALT/AST levels, TNF- $\alpha$, and nitrotyrosine yield are expressed as mean \pm standard deviation, and the Shapiro-Wilk test was used to compare these values in both groups. The significance level was set at $\mathrm{p}<0.05$.

\section{Results \\ Hematology test}

Serum AST/ALT levels were $3085 \pm 436 / 2697 \pm 367$ IU/L in the control group and $857 \pm 97 / 723 \pm 89$ IU/L in the quercetintreated group. TNF- $\alpha$ levels were $7.11 \pm 0.57 \mathrm{pg} / \mathrm{mL}$ in the control group and $5.03 \pm 0.69 \mathrm{pg} / \mathrm{mL}$ in the quercetin-treated group. Nitrotyrosine yield was $0.410 \pm 0.073 \%$ in the control group and $0.050 \pm 0.011 \%$ in the quercetin-treated group. AST/ALT levels, 
TNF- $\alpha$, and nitrotyrosine yield were all significantly lower in the quercetin-treated group (Table 1).

\section{Liver histology findings}

In the control group, hepatic microcirculatory disturbance, liver cell degeneration and necrosis, and focal hemorrhage were observed (Figure 1). In the quercetin-treated group, liver cell structure was maintained, and findings were insignificant (Figure 2).

Table 1: Comparison of serum AST/ALT levels, TNF- $\alpha$, and nitrotyrosine yield in the control and quercetin-treated groups.

\begin{tabular}{|c|c|c|c|}
\hline & Control group & $\begin{array}{c}\text { Quercetin-treated } \\
\text { group }\end{array}$ & p-value \\
\hline AST (IU/L) & $3085 \pm 436$ & $857 \pm 97$ & $<0.01$ \\
\hline ALT (IU/L) & $2697 \pm 367$ & $723 \pm 89$ & $<0.01$ \\
\hline TNF- $\boldsymbol{\alpha}(\mathbf{p g} / \mathbf{m L})$ & $7.11 \pm 0.57$ & $5.03 \pm 0.69$ & $<0.01$ \\
\hline $\begin{array}{c}\text { Nitrotyrosine } \\
(\%)\end{array}$ & $0.410 \pm 0.073$ & $0.050 \pm 0.011$ & $<0.01$ \\
\hline
\end{tabular}

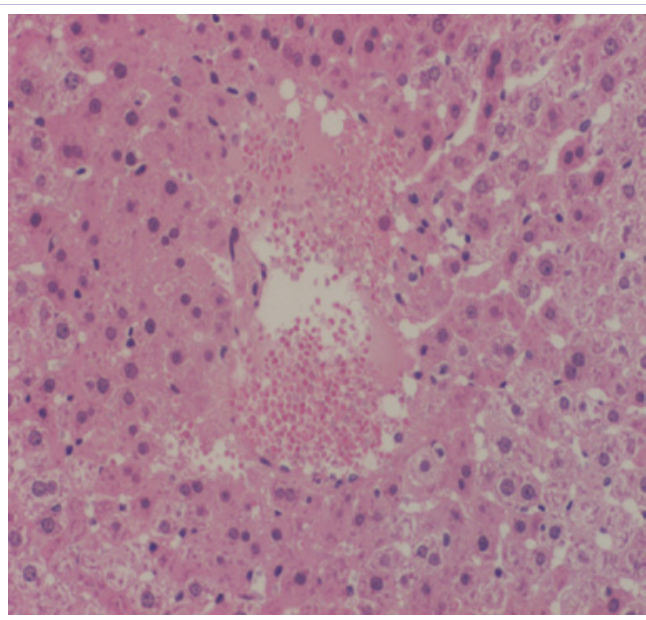

Figure 1: Control group.

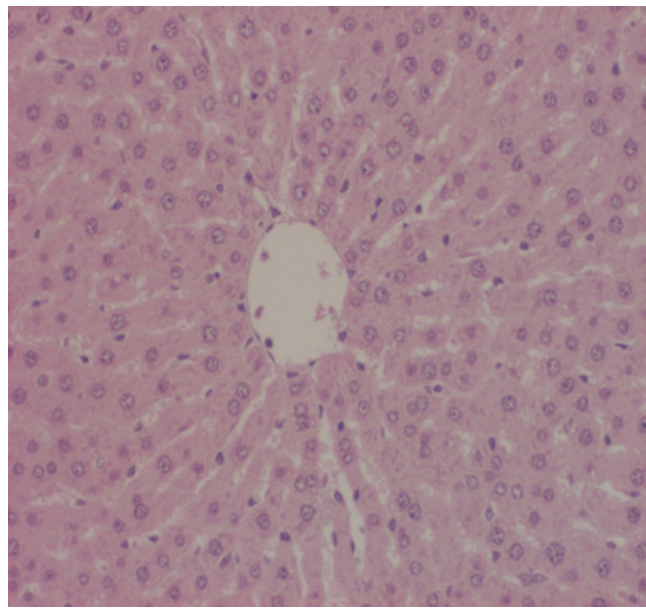

Figure 2: Quercetin-treated group

\section{Discussion}

Previously, both oral [5] and intramuscular administration $[4,8]$ of quercetin has been reported to reduce I/R-induced liver damage in animal models. In our study, intraperitoneal administration of quercetin showed a similar relieving effect on I/R injury.

I/R-induced liver damage occurs in two phases [1]. In the early phase within $2 \mathrm{~h}$ of reperfusion, ROS and cytokines such as TNF- $\alpha$ are significantly involved in liver damage [1-3]. Our study involved blood and histological examination $1 \mathrm{~h}$ after initiating reperfusion; thus, the protective effect of quercetin was examined in the early phase. However, the significant decrease in nitrotyrosine yield and TNF- $\alpha$ suggested that quercetin had an antioxidant and cytokine suppression effect.

Nitrotyrosine is a good indicator of oxidative stress, and two pathways are known to be involved in its production. The first is the nitration of tyrosine residues by peroxynitrite, which is the product of a reaction between superoxides and nitric oxide (NO) $[1,7]$. The second is nitration through the catalytic reaction of myeloperoxidase (MPO) and nitrite, which is a metabolite of NO [12]. Therefore, in the present study, the mechanism underlying the significant decrease in nitrotyrosine yield in the quercetintreated group may be explained by several pathways, as follows:

1) Quercetin has a ROS scavenger effect that may lead to the scavenging of superoxides [4-6,8] 2) Some studies have reported that quercetin inhibits NO production $[2,10,11,13]$. Further, because NO has a vasodilatory effect, it may also have a protective effect on I/R-induced hepatic microcirculatory disturbance [1]. However, because peroxynitrite produced by the reaction between NO and superoxides is highly detrimental to tissues $[1,7]$, the suppression of NO may reduce tissue damage. 3) Quercetin is also reported to have an inhibitory effect on MPO activity [4]; thus, the administration of quercetin may inhibit MPO activity.

In addition, after $I / R$, a rapid increase in blood levels of TNF- $\alpha$ occurs and causes hepatocellular damage and hepatic microcirculatory disturbance as a result of its cytotoxic effect $[1,3,4]$. In the present study, quercetin significantly decreased blood levels of TNF- $\alpha$, which may have contributed to the alleviation of I/R-induced liver damage in the early phase $[1,14]$.

\section{Conclusion}

Our study result demonstrates that quercetin treatment reduces I/R-induced liver damage possibly through both antioxidant and cytokine inhibitory pathways.

\section{Acknowledgements}

The experimental procedures were approved by the Animal Experimentation Committee, School of Medicine, Tokai University, Japan.

This article did not receive specific grants from any funding agencies in the public, commercial, or not-for-profit sectors. 


\section{References}

1. Hines IN, Grisham MB (2011) Divergent roles of superoxide and nitric oxide in liver ischemia and reperfusion injury. J Clin Biochem Nutr 48(1): 50-56

2. Mukhopadhyay P, Rajesh M, Horváth B, Bátkai S, Park O, et al. (2011) Cannabidiol protects against hepatic ischemia/reperfusion injury by attenuating inflammatory signaling and response, oxidative/ nitrative stress, and cell death. Free Radic Biol Med 50(10): 13681381.

3. Papadopoulos D, Siempis T, Theodorakou E, Tsoulfas G (2013). Hepatic ischemia and reperfusion injury and trauma: current concepts. Arch Trauma Res 2(2): 63-70.

4. Polat C, Tokyol C , Kahraman A, Sabuncuoğlu B, Yilmaz S (2006) The effects of desferrioxamine and quercetin on hepatic ischemiareperfusion induced renal disturbance. Prostaglandins Leukot Essent Fatty Acids 74(6): 379-383.

5. Su JF, Guo CJ, Wei JY, Yang JJ, Jiang YG, et al. (2003) Protection against hepatic ischemia-reperfusion injury in rats by oral pretreatment with quercetin. Biomed Environ Sci 16(1): 1-8.

6. Barteková M, Čarnická S, Pancza D, Ondrejčáková M, Breier A, et al. (2010) Acute treatment with polyphenol quercetin improves postischemic recovery of isolated perfused rat hearts after global ischemia. Can J Physiol Pharmacol 88(4): 465-471.

7. Ghosh A, Sarkar S, Mandal AK, Das N (2013) Neuroprotective role of nanoencapsulated quercetin in combating ischemia-reperfusion induced neuronal damage in young and aged rats. PLoS One 8(4): e57735.
8. Tokyol C, Yilmaz S, Kahraman A, Cakar H, Polat C (2006) The effects of desferrioxamine and quercetin on liver injury induced by hepatic ischaemia-reperfusion in rats. Acta Chir Belg 106(1): 68-72.

9. Jin HB, Yang YB, Song YL, Zhang YC, Li YR (2012) Protective roles of quercetin in acute myocardial ischemia and reperfusion injury in rats. Mol Biol Rep 39(12): 11005-11009.

10. Kinaci MK, Erkasap N, Kucuk A, Koken T, Tosun M (2012) Effects of quercetin on apoptosis, NF-кB and NOS gene expression in renal ischemia/reperfusion injury. Exp Ther Med 3(2): 249-254.

11. Endale M, Park SC, Kim S, Kim SH, Yang Y, et al. (2013) Quercetin disrupts tyrosine-phosphorylated phosphatidylinositol 3-kinase and myeloid differentiation factor-88 association, and inhibits MAPK/ AP-1 and IKK/NF-кB-induced inflammatory mediators production in RAW 264.7 cells. Immunobiology 218(12): 1452-1467.

12. Ichimori K, Fukuyama N, Nakazawa $H$, Aratani $Y$, Koyama $H$, et al. (2003) Myeloperoxidase has directly-opposed effects on nitration reaction--Study on myeloperoxidase-deficient patient and myeloperoxidase-knockout mice. Free Radic Res 37(5): 481-489.

13. Wan LL, Xia J, Ye D, Liu J, Chen J, et al. (2009) Effects of quercetin on gene and protein expression of NOX and NOS after myocardial ischemia and reperfusion in rabbit. Cardiovasc Ther 27(1): 28-33.

14. Yuan GJ, Ma JC, Gong ZJ, Sun XM, Zheng SH, et al. (2005) Modulation of liver oxidant-antioxidant system by ischemic preconditioning during ischemia/reperfusion injury in rats. World J Gastroenterol 11(12): 1825-1828. 